\title{
Efficacy of Nucleotide/Nucleoside Analogues and Hepatitis B Immunoglobulin Therapy in Blocking Mother-to-Child Transmission of Hepatitis B in an Eastern Chinese Group
}

\author{
Xiaojun Sun, ${ }^{1}$ Chengwei Wang, ${ }^{2}$ Bian Wang, ${ }^{2}$ Xiuzhen Yang, ${ }^{2}$ Hongtao Xu, ${ }^{2}$ \\ Meilong Shen $\mathbb{D}^{3},{ }^{3}$ and Kuichun Zhu $\mathbb{D}^{4}$ \\ ${ }^{1}$ Taizhou Hospital of Chinese Traditional Medicine, Multidisciplinary Center, Taizhou, Jiangsu 225300, China \\ ${ }^{2}$ Taizhou People's Hospital, Department of Hepatology, Taizhou, Jiangsu 225300, China \\ ${ }^{3}$ Taizhou Hospital of Chinese Traditional Medicine, Department of Gastroenterology \& Hepatology, Taizhou, Jiangsu 225300, China \\ ${ }^{4}$ Wuxi Shenrui Bio-Pharmaceuticals Co., Ltd., R\&D Department, Wuxi, Jiangsu 214000, China
}

Correspondence should be addressed to Meilong Shen; shenm19326@126.com and Kuichun Zhu; kzhu68@hotmail.com

Received 15 May 2020; Revised 19 October 2020; Accepted 24 November 2020; Published 17 December 2020

Academic Editor: Susan Cu-Uvin

Copyright ( 2020 Xiaojun Sun et al. This is an open access article distributed under the Creative Commons Attribution License, which permits unrestricted use, distribution, and reproduction in any medium, provided the original work is properly cited.

\begin{abstract}
The objective of this study was to investigate the efficacy and potential side-effects of nucleotide/nucleoside analogues and hepatitis B immunoglobulin injection of newborns in blocking mother-to-child transmission of hepatitis B virus in the middle and late pregnancy period. 238 cases of enrolled pregnant women were divided into the Telbivudine group, the Tenofovir group, the Lamivudine group, and the hepatitis B immunoglobulin (HBIG) group. Enrolled patients received corresponding therapies. Clinical and laboratory data were collected. Results showed that the levels of HBV DNA of the enrolled pregnant women in the Telbivudine, Tenofovir, and Lamivudine groups decreased rapidly after 12 weeks of drug intervention compared with those in the control. HBsAg positive rate in newborns and in children 24 weeks after birth was $0 / 60,0 / 60,0 / 60,3 / 30$, and $11 / 28$ in the Telbivudine, Tenofovir, Lamivudine, HBIG, and control groups, respectively. No significant side-effects were identified after following up to 12 months after birth. Our results show that routine HBV vaccine plus HBIG injections is insufficient in blocking mother-to-child HBV transmission. Administration of nucleotide/nucleoside analogues or HBIG at pregnancy is suggested to maximize the blocking of vertical HBV transmission.
\end{abstract}

\section{Introduction}

The hepatitis B virus (HBV) infection remains a major health problem in China and worldwide. Infant infection from pregnant women as hepatitis B carriers is still a major concern [13]. For mothers with HBV infection in China, newborns are injected with HBIG and hepatitis $B$ vaccine to interrupt mother-to-child transmission of HBV. However, mother-tochild transmission of HBV still accounts for about 30\% to 50\% new HBV infection [4]. Researches had shown that mother-to-child transmission of HBV mainly occurred in late pregnancy and lactation $[3,5,6]$. nucleotide/nucleoside analogues (NA) have been used in addition to HBIG and HBV vaccine to interrupt vertical transmission of $\mathrm{HBV}$ [7]. To search for more effective approaches in blocking the vertical transmission, we treated pregnant women in middle and late pregnancy period with nucleotide/nucleoside analogues and treated newborns with HBIG to interrupt mother-to-child transmission of $\mathrm{HBV}$, and our results added more thoughts to the prevention strategies of mother-to-child transmission.

\section{Materials and Methods}

2.1. Subjects, Grouping, and Treatment. With the approval of the Ethics Committee of Taizhou People's Hospital, all the enrolled pregnant women signed the informed consent form. All 238 pregnant women were chronic HBV carriers who received obstetric examination in Taizhou People's Hospital from September 2010 to April 2018. Diagnosis was made according to the diagnostic criteria established in 2010 in 
TABLE 1: Study groups and treatments.

\begin{tabular}{lcc}
\hline Groups & Cases & Treatments \\
\hline Telbivudine group & 60 & Telbivudine $600 \mathrm{mg}$ qd, from 26 gestational weeks to 4 weeks postpartum \\
Tenofovir group & 60 & Tenofovir $300 \mathrm{mg}$ qd, from 26 gestational weeks to 4 weeks postpartum \\
Lamivudine group & 60 & Lamivudine $100 \mathrm{mg}$ qd, from 26 gestational weeks to 4 weeks postpartum \\
HBIG group & 30 & HBIG 200 IU/4 weeks for 3 times from 26 gestational weeks \\
Control group & 28 & No NA or HBIG intervention before birth \\
\hline
\end{tabular}

China [8]. No participant received interferon or NA treatment before the study. The blood HBV titers of the enrolled pregnant women were all greater than $10^{4} \mathrm{IU} / \mathrm{ml}$. Patients with primary liver cancer, other viral hepatitis, fatty hepatitis, alcoholic hepatitis, drug hepatitis, and autoimmune hepatitis were excluded.

After enrollment, patients were divided into the Telbivudine group, the Tenofovir group, the Lamivudine group, the hepatitis B immunoglobulin (HBIG) group, and the control group randomly. The age, height, and body weight of pregnant women in each group were comparable. The details of treatment for each group of pregnant women are shown in Table 1. Alanine aminotransferase, total bilirubin, HBsAg, $\mathrm{HBeAg}$, and HBV DNA were routinely tested after enrollment and reexamined every 12 weeks. At the 12 th gestational week, HBV genotyping and drug resistance were examined. The percentage and absolute value of natural killer (NK) cells in the blood at 12 gestational weeks were also examined.

Newborns were routinely injected with hepatitis B vaccine on the day of birth, and HBIG 200 IU was injected at 2 weeks, 4 weeks, and 12 weeks, respectively. Hepatitis B markers were tested using the cord blood of the newborn and the blood at the age of 24 weeks. Pregnant women and newborns were followed up for at least 1 year after childbirth.

2.2. Laboratory Tests. HBV DNA level was tested by the 7300 real-time qPCR instrument and kit of the American Applied Biosystems company according to the manufacturer's instructions. The Abbott AxSYM reagent and instrument were used as the automatic immunoassay system. The titer of HBsAg was expressed as $S / N$, that is, the ratio of the sample to the negative control. It was considered positive when $S / N \geq 2$. The result of $\mathrm{HBeAg}$ was determined by the ratio of sample value to clinical cutoff value, and it was considered positive when the ratio $\geq 1$. The percentage of total NK cells (CD3-CD16+56+) in lymphocytes was examined with flow cytometry, and the absolute number of NK cells was calculated based on blood white blood cell counts. HBV genotyping and drug resistance mutations were performed with PCR amplification of HBV polymerase region and DNA sequencing with an $\mathrm{ABl} 3100$ sequencing instrument. All laboratory tests were performed with nationally certified test methods and materials.

2.3. Statistical Analysis. The rates between groups were analyzed by the $\chi^{2}$ test, and the quantitative measurement data were analyzed by a two-sample $t$-test. Statistical analysis was carried out by the SPSS16.0 software. $P<0.05$ was considered as statistically significant.

\section{Results}

The levels of HBV DNA of the enrolled pregnant women in the Telbivudine, Tenofovir, and Lamivudine groups decreased rapidly after 12 weeks of NA treatment, compared with those in the control group, $P<0.05$ (Table 2). The decrease in HBV levels in the HBIG group was limited (Table 2). There were $2 / 60,1 / 60,1 / 60,4 / 30$, and $12 / 28$ HBsAg-positive newborn cases in the Telbivudine, Tenofovir, Lamivudine, HBIG, and control groups, respectively, at birth. Reexamined at 24 weeks after birth, there were $0 / 60$, $0 / 60,0 / 60,3 / 30$, and 11/28 newborn cases of HBsAg positivity in the Telbivudine, Tenofovir, Lamivudine, HBIG, and control groups, respectively, $P<0.05$ (Table 3 ).

There was no severe adverse reaction during the treatment period in the treatment groups. Transient mild increase in serum creatine kinase was found in 1 case in the Telbivudine group and 1 case in the Lamivudine group; the level returned to normal after two weeks. There was no significant difference in gestational age, mean weight, and Apgar score among all groups (Table S1). There were no complications such as fetal distress, neonatal asphyxia, amniotic fluid contamination, abnormal development, or malformations in each group. When the infants in each group were followed up to the age of 12 months, no deformity or other abnormalities were found.

All the patients enrolled in this study carried HBV genotype B or C. Our results showed that $\mathrm{HBsAg}, \mathrm{HBeAg}$, genotyping, $\mathrm{HBV}$ drug resistance, or the number of NK cells was not significantly correlated with treatment outcomes in patients (Table S2).

\section{Discussion}

Mother-to-child transmission is still a main transmission route of $\mathrm{HBV}$ infection in China. Over $90 \%$ of the newborns who get infected HBV develop into chronic hepatitis B carrier status $[9,10]$. Infants infected by mother-to-child transmission could carry HBV for dozens of years [4]. High HBV DNA level and abnormal liver function lead to high motherto-child transmission rates and adverse pregnancy events [11]. At present, active and passive immune intervention still could not completely interrupt the transmission from mother to child, with about $9 \%$ newborns from HBV carrier mothers still getting infected [12]; the infection rate could be around $40 \%$ if no intervention is provided [13].

High HBV DNA level can easily lead to placental dysplasia which makes the trophoblast cells of the placenta lose the protective barrier function, resulting in transplacental 
TABLE 2: The blood HBV DNA levels in study subjects.

\begin{tabular}{lcc}
\hline Group & Before treatment & 12 weeks after treatment \\
\hline Telbivudine group & $6.69 \pm 1.72$ & $2.18 \pm 1.35^{\#}$ \\
Tenofovir group & $6.72 \pm 1.56$ & $2.26 \pm 1.40^{\#}$ \\
Lamivudine group & $6.80 \pm 1.59$ & $2.34 \pm 1.44^{\#}$ \\
HBIG group & $6.77 \pm 1.51$ & $5.17 \pm 1.83$ \\
Control group & $6.72 \pm 1.64$ & $6.80 \pm 1.56$ \\
\hline
\end{tabular}

Numbers were $M \pm \mathrm{SD}$ of the group at $\log$ scale. ${ }^{\#}$ Statistical significance compared with the control group, $P<0.05$.

TABLE 3: HBsAg status in newborns.

\begin{tabular}{lccc}
\hline Groups & Cases & $\begin{array}{c}\text { HBsAg+ } \\
\text { cases at birth }\end{array}$ & $\begin{array}{c}\text { HBsAg+ cases 24 } \\
\text { weeks after birth }\end{array}$ \\
\hline Telbivudine group & 60 & 2 & $0^{\# *}$ \\
Tenofovir group & 60 & 1 & $0^{\# *}$ \\
Lamivudine group & 60 & 1 & $0^{\# *}$ \\
HBIG group & 30 & 4 & $3^{\#}$ \\
Control group & 28 & 12 & 11 \\
\hline
\end{tabular}

${ }^{\#}$ Statistical significance compared with the control group, $P<0.05$. *Statistical significance compared with the HBIG group, $P<0.05$.

hematogenous and cell-derived intrauterine infection $[4,7]$. Therefore, reducing HBV DNA level is considered the key to restore the placental barrier and reduce intrauterine transmission of HBV $[14,15]$. Recent studies had shown that Telbivudine, Tenofovir, and Lamivudine could effectively reduce the HBV DNA level in pregnant women [7, 16]. Our study showed that HBV DNA titers of pregnant women in the Telbivudine, Tenofovir, and Lamivudine groups were significantly decreased after 12 weeks of treatment, consistent with the literature [17]. At 24 weeks after birth, the HBsAg positive rate was $0 / 60,0 / 60,0 / 60,3 / 30$, and $11 / 28$ in the Telbivudine, Tenofovir, Lamivudine, HBIG, and control groups, respectively. The significant differences indicate that the interruption approaches at pregnancy were effective in blocking HBV transmission. HBIG is a concentrated anti-HBV passive immune protection agent [18]. HBIG can activate the complement system, enhance humoral immunity, and significantly reduce the HBV load in pregnant women [19]. Our results showed that 3 of the 30 newborns in the HBIG group tested HBsAg positive at 24 weeks after birth. Compared with the HBIG group, the Telbivudine, Tenofovir, and Lamivudine groups showed stronger inhibition of HBV. Genotype, drug resistance, absolute value of NK cells, $\mathrm{HBsAg}$, and $\mathrm{HBeAg}$ in pregnant women with hepatitis B carriers did not correlate with treatment outcomes.

Early pregnancy is the most important period of infant development growth, so the safety of Telbivudine, Tenofovir, and Lamivudine was of great concern [17, 20]. Telbivudine and Tenofovir are class B drugs for pregnancy, and Lamivudine is a class $\mathrm{C}$ drug for pregnancy. Consistent with the literature reports, our results showed that Telbivudine, Tenofovir, and Lamivudine were relatively safe $[21,22]$.
In summary, our results showed that administration of nucleotide/nucleoside analogues Telbivudine, Tenofovir, and Lamivudine and, to a lesser extent, the sequential hepatitis B immunoglobulin injections in pregnant women were effective in blocking mother-to-child transmission of hepatitis virus. Routine $\mathrm{HBV}$ vaccine plus HBIG injections was insufficient in blocking mother-to-child HBV transmission. Administration of nucleotide/nucleoside analogues or HBIG at pregnancy is suggested to maximize the blocking of vertical HBV transmission.

\section{Data Availability}

All data generated or analyzed in this study are included in the article and its supplementary information files.

\section{Conflicts of Interest}

The authors declare no conflicts of interest.

\section{Authors' Contributions}

Xiaojun Sun and Chengwei Wang contributed equally to the work.

\section{Acknowledgments}

The work was supported in part by NSF of Jiangsu Province for basic research (BK2011538, SML).

\section{Supplementary Materials}

Table S1: characteristics of newborns. Table S2: other characteristics of subjects in each group. (Supplementary Materials)

\section{References}

[1] N. A. Terrault, A. S. F. Lok, B. J. McMahon et al., "Update on prevention, diagnosis, and treatment of chronic hepatitis B: AASLD 2018 hepatitis B guidance," Hepatology, vol. 67, no. 4, pp. 1560-1599, 2018.

[2] I. D. Batsis, P. Wasuwanich, and W. W. Karnsakul, "The management of hepatitis B and hepatitis C in children," Minerva Pediatrica, vol. 71, no. 1, pp. 59-75, 2019.

[3] C. E. Stevens, P. Toy, S. Kamili et al., "Eradicating hepatitis B virus: the critical role of preventing perinatal transmission," Biologicals, vol. 50, pp. 3-19, 2017.

[4] Z. Han, Y. Yin, Y. Zhang et al., "Knowledge of and attitudes towards hepatitis B and its transmission from mother to child among pregnant women in Guangdong Province, China," PLoS One, vol. 12, no. 6, article e0178671, 2017.

[5] M. J. Tong, C. Q. Pan, S. H. B. Han et al., “An expert consensus for the management of chronic hepatitis B in Asian Americans," Alimentary Pharmacology \& Therapeutics, vol. 47, no. 8, pp. 1181-1200, 2018.

[6] J. Li, M. S. Chang, T. T. Tran, and M. H. Nguyen, "Management of chronic hepatitis B in pregnancy," Journal of Clinical Gastroenterology, vol. 51, no. 9, pp. 789-795, 2017.

[7] S. Tavakolpour, M. Darvishi, H. S. Mirsafaei, and M. Ghasemiadl, "Nucleoside/nucleotide analogues in the treatment of chronic hepatitis B infection during pregnancy: a 
systematic review," Infectious Diseases, vol. 50, no. 2, pp. 95106, 2018.

[8] S. Manzoor, M. Saalim, M. Imran, S. Resham, and J. Ashraf, "Hepatitis B virus therapy: what's the future holding for us?," World Journal of Gastroenterology, vol. 21, no. 44, pp. 12558-12575, 2015.

[9] S. S. Joshi and C. S. Coffin, "Hepatitis B and pregnancy: virologic and immunologic characteristics," Hepatology Communications, vol. 4, no. 2, pp. 157-171, 2019.

[10] S. K. Sarin, M. Kumar, G. K. Lau et al., "Asian-Pacific clinical practice guidelines on the management of hepatitis B: a 2015 update," Hepatology International, vol. 10, no. 1, pp. 1-98, 2016.

[11] P. A. Revill, F. V. Chisari, J. M. Block et al., "A global scientific strategy to cure hepatitis B," The Lancet Gastroenterology \& Hepatology, vol. 4, no. 7, pp. 545-558, 2019.

[12] Y. Hu and H. Yu, "Prevention strategies of mother-to-child transmission of hepatitis B virus (HBV) infection," Pediatric Investigation, vol. 4, no. 2, pp. 133-137, 2020.

[13] C. L. Lin and J. H. Kao, "Review article: the prevention of hepatitis B-related hepatocellular carcinoma," Alimentary Pharmacology \& Therapeutics, vol. 48, no. 1, pp. 5-14, 2018.

[14] X. Gao, X. Duan, H. Cai et al., "Pregnancy outcomes for pregnant women with chronic hepatitis B exposing to entecavir or adefovir dipivoxil therapy before or in early pregnancy," The Journal of Maternal-Fetal \& Neonatal Medicine, pp. 1-5, 2020.

[15] Q. J. Sheng, S. J. Wang, Y. Y. Wu, X. G. Dou, and Y. Ding, "Hepatitis B virus serosurvey and awareness of mother-tochild transmission among pregnant women in Shenyang, China: an observational study," Medicine (Baltimore), vol. 97, no. 22, article e10931, 2018.

[16] M. Makvandi, "Update on occult hepatitis B virus infection," World Journal of Gastroenterology, vol. 22, no. 39, pp. 87208734, 2016.

[17] T. Piratvisuth, G. R. Han, S. Pol, Y. Dong, and A. Trylesinski, "Comprehensive review of telbivudine in pregnant women with chronic hepatitis B," World Journal of Hepatology, vol. 8, no. 9, pp. 452-460, 2016.

[18] Y. Zhao, H. Jin, X. Zhang, B. Wang, and P. Liu, "Viral hepatitis vaccination during pregnancy," Human Vaccines \& Immunotherapeutics, vol. 12, no. 4, pp. 894-902, 2016.

[19] E. Ç. Tanrıverdi, Z. Özkurt, B. G. Kadıŏglu et al., "Seroprevalence of hepatitis $\mathrm{B}$, hepatitis $\mathrm{C}$, and HIV in pregnant women from eastern Turkey," The Turkish Journal of Gastroenterology, vol. 30, no. 3, pp. 260-265, 2019.

[20] K. Zhou and N. Terrault, "Management of hepatitis B in special populations," Best Practice \& Research. Clinical Gastroenterology, vol. 31, no. 3, pp. 311-320, 2017.

[21] S. Sali, M. Darvishi, M. GhasemiAdl et al., "Comparing the efficacy and safety of treating chronic hepatitis B infection during pregnancy with lamivudine, telbivudine, and tenofovir: a meta-analysis," Journal of Clinical and Translational Hepatology, vol. 7, no. 3, pp. 197-212, 2019.

[22] Q. Sheng, Y. Ding, B. Li et al., "Efficacy and safety of nucleos(t)ide analogues to prevent hepatitis B virus mother-to-child transmission in pregnant women with high viremia: real life practice from China," International Journal of Medical Sciences, vol. 15, no. 8, pp. 796-801, 2018. 